\title{
Enhancement of chitosan-graphene oxide SPR sensor with a multi-metallic layers of Au-Ag-Au nanostructure for lead(II) ion detection
}

\begin{abstract}
We demonstrate the enhancement of surface plasmon resonance (SPR) technique by implementing a multi-metallic layers of Aul Agï Au nanostructure in the chitosan-graphene oxide (CS-GO) SPR sensor for lead(II) ion detection. The performance of the sensor is analyzed via SPR measurements, from which the sensitivity, signal-to-noise ratio and repeatability are determined. The nanostructure layers are characterized using field emission scanning electron microscopy (FESEM), atomic force microscopy (AFM), X-ray diffraction (XRD), Raman spectroscopy and X-ray photoelectron spectroscopy (XPS). We showed that the proposed structure has increased the shift in the SPR angle up to $3.5^{\circ}$ within the range of 0.11 i $1 \mathrm{ppm}$ due to the enhanced evanescent field at the sensing layer-analyte interface. This sensor also exhibits great repeatability which benefits from the stable multi-metallic nanostructure. The SNR value of 0.92 for $5 \mathrm{ppm}$ lead(II) ion solution and reasonable linearity range up to that concentration shows that the tri-metallic CS-GO SPR sensor gives a good response towards the lead(II) ion solution. The CS-GO SPR sensor is also sensitive to at least a 10i 5 change in the refractive index. The results prove that our proposed tri-metallic CS-GO SPR sensor demonstrates a strong performance and reliability for lead(II) ion detection in accordance with the standardized lead safety level for wastewater.
\end{abstract}

Keyword: Surface plasmon resonance; Multi-metallic; Nanostructured; Chitosan; Graphene oxide; Lead(II) ion detection 